\title{
The Speed of the Passing of Time as Yet Another Facet of Cosmic Dark Energy
}

\section{—Dedicated to Prof. J.M.T. Thompson, FRS in Reminiscence of the Good Old Times}

\author{
M. S. El Naschie \\ Department of Physics, Faculty of Science, Alexandria University, Alexandria, Egypt \\ Email: Chaossf@aol.com
}

How to cite this paper: El Naschie, M.S. (2016) The Speed of the Passing of Time as Yet Another Facet of Cosmic Dark Energy. Journal of Modern Physics, 7, 2103-2125. http://dx.doi.org/10.4236/jmp.2016.715184

Received: November 3, 2016 Accepted: November 7, 2016

Published: November 10, 2016

Copyright $\odot 2016$ by author and Scientific Research Publishing Inc. This work is licensed under the Creative Commons Attribution International License (CC BY 4.0).

http://creativecommons.org/licenses/by/4.0/

\begin{abstract}
The topological speed of light which may be used to compute the density of ordinary energy and dark energy of the cosmos is replaced by dimensionless quantity taken from Special Relativity. The said quantity may be interpreted as akin to time dilation ergo a notion topologically equivalent to the speed of the passing of time or the difference of elapsed time between two events in Einstein's Relativity Theory. This results via Newton's kinetic energy into the well-known observationally confirmed and accurately measured 4.5 and 95.5 percent of ordinary and dark Cosmic Energy density respectively.
\end{abstract}

\section{Keywords}

Topological Speed of Light, Speed of Passing of Time, Dark Energy, Einstein Special Relativity, E-Infinity Theory, Cantorian Spacetime

\section{Introduction}

"Time passes. Listen time passes ..." These were some of the most haunting words the Author encountered as a young man when he was (and still is) fascinated by both Dylan Thomas' Under Milk Wood [1] and the acting and voice of Richard Burton in the identically entitled film [2]. At the time I was privileged to be a Ph.D. student of Prof. J. M. T. Thompson, FRS at University College, London [3]. Although working in a Civil Engineering Department [3] [4] both of us were mainly interested in fundamental science, specially Relativity. Apart of that we had deep affinity with Literature and Modern music particularly Dylan Thomas [1] [2] and Bob Dylan [5]. Nevertheless the Author must admit that neither he nor Michael Thompson ever suspected any scientific association between the psychological phenomenon of the passing of time [6] and the 
mathematical relation of the same subject in Einstein's Special Relativity let alone any connection whatsoever to the Cosmic ordinary and dark energy density of the Universe. This field of scientific inquiry was yet to be discovered many decades later and was investigated and analyzed using the present Author's E-Infinity Cantorian Spacetime theory which generalized Special and General Relativity and unified them with Quantum Mechanics [7]-[427].

In the present paper, we will attempt to describe the genesis of the concept behind the topological speed of light, which was used to compute the cosmological energy density [27] [165], to become this mathematical concept of the speed of the passing of time which is used here for the same purpose [7] [29]. Remarkably but not unexpectedly both concepts give identical results [7] [29] [165]. It is not our intent to plea for one concept or the other as more natural or easier to grasp but we hope that the present wok increases the confidence into the reality of the Dark Energy of the Quantum Wave [197] and the solid correctness of our quantitative analytically obtained results which is in superb agreement with all cosmic measurements and observations [89] [140]. Needless to say that, such agreement on its own account alone makes our theory almost refutation proof.

\section{The Essence of the Topological Speed of Light in E-Infinity Theory}

Motivated by the irreducible discreteness of quantum mechanics [8]-[28] and the mathematical-logic inconsistency in the definition of a classical point, a "pointless" Cantorian spacetime was advanced [15] [74] [420]. In this meantime well-known approach, which is based on previous work by Von Neumann and A. Connes [420], a classical point was practically replaced by an entire random Cantor set [8] [10] [420]. Such set is naturally of a Zero measure and Zero topological dimension [22] [33] [100]. Thus the only non Zero quantity attached to this set is a Hausdorff dimension equal to the golden mean $\phi$ as per the Von Neumann-Connes dimensional function [10] [420]. The result of such consistent but truly drastic geometrical simplification was that a subtle and delicate equality took place by which both the Hausdorff dimension $\phi$ became also the only representative of the "spirit" of the speed of light whereby one may set $\mathrm{c}$ to become $\mathrm{c}=\phi$ [33]. The highly mathematical but simple argument leading to this unexpected result was explained elsewhere and is based on the Transfinite Set theory and Ultimate L [33] [48] [74] as applied to the unit interval of a Clopen random cantor set being the building block of E-Infinity Cantorian Spacetime. This fractal-like transfinite spacetime effectively extended the Minkowsky-Einstein spacetime program [8] [13] [41] [66] to encompass all scales i.e. from the large scale of the cosmos to the quantum scale of elementary particles [8] [10] [33] [74]. Taking now the kinetic energy density of Newton to the Cantorian Penrose-Connes universe one finds that [74] [79] [100]

$$
\mathrm{E}(\text { kinetic })=\frac{1}{2} \mathrm{mv}^{2}
$$


where $\mathrm{m}$ is the mass of a particle and $\mathrm{V}$ is its speed transfer via $\mathrm{v} \rightarrow \mathrm{c}=\phi$ to [74] [100]

$$
\mathrm{E}=\frac{1}{2} \mathrm{~m} \phi^{2}
$$

where $\mathrm{C}$ is the speed of Light and

$$
\phi=(\sqrt{5}-1) / 2
$$

Noting further that $\mathrm{m}$ is $\mathrm{m}=\phi^{3}$ for ordinary energy while $\mathrm{m}=5$ for dark energy [81] [112], one finds the well known final results [138] [140]

$$
\mathrm{E}(\mathrm{O})=\phi^{5} / 2
$$

for dimensionless ordinary energy and [138] [139] [140]

$$
\mathrm{E}(\mathrm{D})=\left(5 \phi^{2} / 2\right)
$$

for dimensionless dark energy [74]. Subsequently a simple multiplication of the above with Einstein's density $\mathrm{E}=\mathrm{mc}^{2}$ gives us the final results namely [84] [85]

$$
\begin{aligned}
\mathrm{E}(\mathrm{O})= & \left(\phi^{5} / 2\right) \mathrm{mc}^{2} \\
& =\mathrm{mc}^{2} / 22+\mathrm{k} \\
& \simeq \mathrm{mc}^{2} / 22 \\
& \simeq 4.5 \%
\end{aligned}
$$

and [85]

$$
\begin{aligned}
\mathrm{E}(\mathrm{D}) & =\left(5 \phi^{2} / 2\right) \mathrm{mc}^{2} \\
& \simeq \mathrm{mc}^{2}(21 / 22) \\
& \simeq 95.5 \%
\end{aligned}
$$

The above result is in full agreement with a dozen or so previous derivations as well as being in more than excellent agreement with all modern recent measurements and observations [25] [27].

It is instructive to note that $\phi^{5}$ may be interpreted as multiplicative topological volume of a five-dimensional Zero set quantum particle while $5 \phi^{2}$ is the additive topological volume of a given dimensional empty set quantum wave [33] [81].

\section{From the Speed of Passing of Time to Dark Energy}

Although time does not pass for Albert Einstein as documented in his letters to the widow of his best friend M. Besso [427] there are some beautiful formulas and controversial interpretations of many consequences of Special Relativity which have bearing on this issue and the present work [33] [34] [236]. Taking things at their face value, it is possible to deduce following Ref. [7] that

$$
\mathrm{t}^{1} / \mathrm{t}=\frac{1-(\mathrm{v} / \mathrm{c})}{\sqrt{1-\left(\mathrm{v}^{2} / \mathrm{c}^{2}\right)}}
$$

It is important to note that this formula is similar but not identical to the well-known 
time dilation of Einstein's Special Relativity Theory. In particular our present results are correct only for $\mathrm{c} / \mathrm{v}=\sqrt{5}$ as noted in [7].

Furthermore following many previous results mainly due to Sigalotti as well as the members of the E-Infinity Group [27] [28] [29] [30] which clearly motivated the recent paper by the Serbian Mathematician C. Jozsef [7], it is possible to prove that $t^{1} / t=\phi$ as shown in Ref. [7]. In terms of E-Infinity theory and our preceding discussion we could say that the speed of the passing of time is identical to the topological speed of light and consequently $\mathrm{c}=\phi$ [7] [33] [34] [35]. Adding $\mathrm{m}(\mathrm{O})=\phi^{3}$ and $\mathrm{m}(\mathrm{D})=5$ to the above, the results of the preceding dissection of $\mathrm{E}=\mathrm{mc}^{2}$ into $\mathrm{E}(\mathrm{O})=\mathrm{mc}^{2} / 22$ and $\mathrm{E}(\mathrm{D})=\mathrm{mc}^{2}(21 / 22)$ follows [66] [85] [236].

\section{Conclusion}

On a realistic mundane level one could say time does not pass. Only we humans pass. However Pure mathematics has normally little patience with the preceding poetic utterance. Nevertheless the mathematical utterance $t^{1} / t=\phi$ given in Ref. [7] and in a different form earlier on by the remarkable Italian Physicist L. Sigalotti [35] has consequence not only for us humans but for the entire universe and its formation and energy density. It is amazing how pure mathematics and mathematical logic can unite the un-unitable such as feeling and facts and all that via the magic of a Golden section based computation [236].

\section{Acknowledgements}

The Author is deeply indebted to his colleagues M. A. Helal, J-Huan He, L. M. Crnjac and L. Sigalotti.

\section{References}

[1] Dylan, T. (1954) Under Milk Wood. New Direction Publishing Corporation, New York.

[2] Sinclair, A. (Director) (1972) Under Milk Wood. The Film. Starring R. Burton, Elizabeth Taylor and P. O’Toole.

[3] Thompson. J.M.T. (1970) University College London, U.K. Google Citation.

[4] El Naschie, M.S. (1975) ZAMM-Journal of Applied Mathematics and Mechanics/Zeitschrift für Angewandte Mathematik und Mechanik, 55, 533-534.

http://dx.doi.org/10.1002/zamm.19750550914

[5] Dylan, B. (2016) Facts. Nobel prize.org Nobel Prize in Literature.

[6] Clark, A. (1998) The Journal of Philosophy, 95, 354-376. http://dx.doi.org/10.2307/2564539

[7] Jozsef, C. (2016) Journal of Modern Physics, 7, 1944-1948. http://dx.doi.org/10.4236/jmp.2016.714171

[8] El Naschie, M.S. (2004) Chaos, Solitons \& Fractals, 19, 209-236. http://dx.doi.org/10.1016/S0960-0779(03)00278-9

[9] El Naschie, M.S. (1995) Quantum Mechanics, Diffusion and Chaotic Fractals. Pergamon.

[10] El Naschie, M.S. (2009) Chaos, Solitons \& Fractals, 41, 2635-2646. 
http://dx.doi.org/10.1016/j.chaos.2008.09.059

[11] El Naschie, M.S. (2006) Chaos, Solitons \& Fractals, 30, 579-605. http://dx.doi.org/10.1016/j.chaos.2006.03.030

[12] El Naschie, M.S. (2006) International Journal of Nonlinear Sciences and Numerical Simulation, 7, 407-409.

[13] El Naschie, M.S. (2007) International Journal of Nonlinear Sciences and Numerical Simulation, 8, 11-20. http://dx.doi.org/10.1515/ijnsns.2007.8.1.11

[14] El Naschie, M.S. (2005) Chaos, Solitons \& Fractals, 24, 1-5. http://dx.doi.org/10.1016/j.chaos.2004.09.001

[15] El Naschie, M.S. (1998) Chaos, Solitons \& Fractals, 9, 931-933. http://dx.doi.org/10.1016/S0960-0779(98)00077-0

[16] El Naschie, M.S. (2006) Chaos, Solitons \& Fractals, 27, 843-849. http://dx.doi.org/10.1016/j.chaos.2005.06.002

[17] El Naschie, M.S. (1997) Chaos, Solitons \& Fractals, 8, 131-133. http://dx.doi.org/10.1016/S0960-0779(96)00128-2

[18] El Naschie, M.S. (1994) Chaos, Solitons \& Fractals, 4, 177-179. http://dx.doi.org/10.1016/0960-0779(94)90141-4

[19] El Naschie, M.S. (1996) Chaos, Solitons \& Fractals, 7, 499-518. http://dx.doi.org/10.1016/0960-0779(96)00007-0

[20] El Naschie, M.S. (2014) International Journal of Astronomy and Astrophysics, 4, 80-90. http://dx.doi.org/10.4236/ijaa.2014.41009

[21] Nottale, L. (1996) Chaos, Solitons \& Fractals, 7, 877-938. http://dx.doi.org/10.1016/0960-0779(96)00002-1

[22] Marek-Crnjac, L., El Naschie, M.S. and He, J.H. (2013) International Journal of Modern Nonlinear Theory and Application, 2, 78. http://dx.doi.org/10.4236/ijmnta.2013.21A010

[23] El Naschie, M.S. (2005) Chaos, Solitons \& Fractals, 25, 969-977. http://dx.doi.org/10.1016/j.chaos.2005.02.028

[24] El Naschie, M.S. (2013) Journal of Quantum Information Science, 3, 23. http://dx.doi.org/10.4236/jqis.2013.31006

[25] El Naschie, M.S. (2013) International Journal of Astronomy and Astrophysics, 3, 205. http://dx.doi.org/10.4236/ijaa.2013.33024

[26] El Naschie, M.S. (2005) International Journal of Nonlinear Sciences and Numerical Simulation, 6, 331-333.

[27] El Naschie, M.S. (2013) International Journal of Astronomy and Astrophysics, 3, 483-493. http://dx.doi.org/10.4236/ijaa.2013.34056

[28] He, J.H. (2005) International Journal of Nonlinear Sciences and Numerical Simulation, 6, 343-346. http://dx.doi.org/10.1515/IJNSNS.2005.6.4.343

[29] El Naschie, M.S. (2013) International Journal of Modern Nonlinear Theory and Application, 2, 43. http://dx.doi.org/10.4236/ijmnta.2013.21005

[30] El Naschie, M.S. (2013) Journal of Quantum Information Science, 3, 121-126. http://dx.doi.org/10.4236/jqis.2013.34016

[31] He, J.H. (2005) International Journal of Nonlinear Sciences and Numerical Simulation, 6, 93-94. http://dx.doi.org/10.1515/IJNSNS.2005.6.2.93

[32] El Naschie, M.S. (2004) Chaos, Solitons \& Fractals, 20, 917-923. http://dx.doi.org/10.1016/j.chaos.2003.11.001 
[33] El Naschie, M.S. (2013) Journal of Modern Physics, 4, 591. http://dx.doi.org/10.4236/jmp.2013.45084

[34] El Naschie, M. (2014) World Journal of Mechanics, 4, Article ID: 47445.

[35] Sigalotti, L.D.G. and Mejias, A. (2006) International Journal of Nonlinear Sciences and Numerical Simulation, 7, 467-472. http://dx.doi.org/10.1515/IJNSNS.2006.7.4.467

[36] El Naschie, M.S. (1995) Chaos, Solitons \& Fractals, 5, 1031-1032. http://dx.doi.org/10.1016/0960-0779(95)00044-5

[37] El Naschie, M.S. (2006) Chaos, Solitons \& Fractals, 29, 876-881. http://dx.doi.org/10.1016/j.chaos.2005.12.027

[38] El Naschie, M.S. (2014) American Journal of Astronomy \& Astrophysics, 2, 72-77. http://dx.doi.org/10.11648/j.ajaa.20140206.13

[39] El Naschie, M.S. (2004) Chaos, Solitons \& Fractals, 19, 1339-1344. http://dx.doi.org/10.1016/j.chaos.2003.08.009

[40] El Naschie, M.S. and Helal, A. (2013) International Journal of Astronomy and Astrophysics, 3, 318. http://dx.doi.org/10.4236/ijaa.2013.33037

[41] Iovane, G. (2005) Chaos, Solitons \& Fractals, 23, 351-360. http://dx.doi.org/10.1016/j.chaos.2004.05.032

[42] El Naschie, M.S. (2014) American Journal of Astronomy \& Astrophysics, 2, No. 3. http://dx.doi.org/10.11648/j.ajaa.20140203.12

[43] El Naschie, M.S. (2007) Chaos, Solitons \& Fractals, 31, 537-547. http://dx.doi.org/10.1016/j.chaos.2006.07.001

[44] El Naschie, M.S. (2006) Chaos, Solitons \& Fractals, 30, 525-531. http://dx.doi.org/10.1016/j.chaos.2005.04.123

[45] El Naschie, M.S. (2013) International Journal of Modern Nonlinear Theory and Application, 2, 107. http://dx.doi.org/10.4236/ijmnta.2013.22014

[46] El Naschie, M.S. (2004) Chaos, Solitons \& Fractals, 21, 249-260. http://dx.doi.org/10.1016/j.chaos.2003.12.001

[47] El Naschie, M.S. (2004) Chaos, Solitons \& Fractals, 20, 437-450. http://dx.doi.org/10.1016/j.chaos.2003.09.029

[48] El Naschie, M.S. (2013) Journal of Quantum Information Science, 3, Article ID: 32831.

[49] El Naschie, M.S. (2015) The Open Astronomy Journal, 8, 1-17. http://dx.doi.org/10.2174/1874381101508010001

[50] El Naschie, M.S. (2006) International Journal of Nonlinear Sciences and Numerical Simulation, 7, 129-132. http://dx.doi.org/10.1515/IJNSNS.2006.7.2.129

[51] El Naschie, M.S. (2014) World Journal of Nuclear Science and Technology, 4, 216. http://dx.doi.org/10.4236/wjnst.2014.44027

[52] El Naschie, M.S. (2013) Journal of Modern Physics, 4, Article ID: 32975. http://dx.doi.org/10.4236/JMP.2013.46103

[53] Tang, W., Li, Y., Kong, H.Y. and El Naschie, M.S. (2014) Bubbfil Nanotechnology, 1, 3-12.

[54] Iovane, G. and Benedetto, E. (2005) International Journal of Nonlinear Sciences and Numerical Simulation, 6, 357-370. http://dx.doi.org/10.1515/IJNSNS.2005.6.4.357

[55] El Naschie, M.S. (2004) Chaos, Solitons \& Fractals, 22, 1-11. http://dx.doi.org/10.1016/j.chaos.2004.01.015

[56] El Naschie, M.S. (2004) Chaos, Solitons \& Fractals, 22, 495-511. 
http://dx.doi.org/10.1016/j.chaos.2004.02.028

[57] El Naschie, M.S. (2006) Chaos, Solitons \& Fractals, 27, 297- 330. http://dx.doi.org/10.1016/j.chaos.2005.04.116

[58] Iovane, G. (2005) Chaos, Solitons \& Fractals, 25, 775-779. http://dx.doi.org/10.1016/j.chaos.2005.02.024

[59] El Naschie, M.S. and Marek-Crnjac, L. (2012) International Journal of Modern Nonlinear Theory and Application, 1, 118. http://dx.doi.org/10.4236/ijmnta.2012.14018

[60] El Naschie, M.S. (2000) Chaos, Solitons \& Fractals, 11, 1149-1162. http://dx.doi.org/10.1016/S0960-0779(99)00185-X

[61] El Naschie, M.S. (2006) Chaos, Solitons \& Fractals, 30, 622-628. http://dx.doi.org/10.1016/j.chaos.2006.04.042

[62] El Naschie, M.S. (2013) Journal of Modern Physics and Applications, 2014, Article-ID: 2.

[63] El Naschie, M.S. (2013) Journal of Modern Physics, 4, 1417. http://dx.doi.org/10.4236/jmp.2013.410170

[64] El Naschie, M.S. (2013) Journal of Modern Physics, 4, 354. http://dx.doi.org/10.4236/jmp.2013.43049

[65] El Naschie, M.S. (2003) Chaos, Solitons \& Fractals, 16, 353-366. http://dx.doi.org/10.1016/S0960-0779(02)00440-X

[66] El Naschie, M.S. (2014) Journal of Quantum Information Science, 4, 284. http://dx.doi.org/10.4236/jqis.2014.44023

[67] El Naschie, M.S. (1993) Journal of the Franklin Institute, 330, 199-211. http://dx.doi.org/10.1016/0016-0032(93)90030-X

[68] El Naschie, M.S. (2003) Chaos, Solitons \& Fractals, 18, 401-420. http://dx.doi.org/10.1016/S0960-0779(03)00098-5

[69] El-Ahmady, A.E. (2007) Chaos, Solitons \& Fractals, 31, 1272-1278. http://dx.doi.org/10.1016/j.chaos.2005.10.112

[70] El Naschie, M.S. (2005) Chaos, Solitons \& Fractals, 24, 447-457. http://dx.doi.org/10.1016/j.chaos.2004.09.071

[71] El Naschie, M.S. (2014) World Journal of Condensed Matter Physics, 4, 74-77. http://dx.doi.org/10.4236/wjcmp.2014.42011

[72] El Naschie, M.S. (2014) Problems of Nonlinear Analysis in Engineering Systems, 20, 79-98.

[73] El Naschie, M.S. (2014) Journal of Quantum Information Science, 4, 83-91. http://dx.doi.org/10.4236/jqis.2014.42008

[74] Helal, M.A., Marek-Crnjac, L. and He, J.H. (2013) Open Journal of Microphysics, 3, 141. http://dx.doi.org/10.4236/ojm.2013.34020

[75] El Naschie, M.S. (2007) Chaos, Solitons \& Fractals, 32, 911-915. http://dx.doi.org/10.1016/j.chaos.2006.08.014

[76] El Naschie, M.S. (2008) Chaos, Solitons \& Fractals, 35, 202-211. http://dx.doi.org/10.1016/j.chaos.2007.05.006

[77] El Naschie, M.S. (2014) Open Journal of Fluid Dynamics, 4, 15-17. http://dx.doi.org/10.4236/ojfd.2014.41002

[78] El Naschie, M.S. (2016) Quantum Matter, 5, 1-4. http://dx.doi.org/10.1166/qm.2016.1247

[79] El Naschie, M.S. (2015) Advances in Pure Mathematics, 5, 560. http://dx.doi.org/10.4236/apm.2015.59052 
[80] El Naschie, M.S. (2007) International Journal of Nonlinear Sciences and Numerical Simulation, 8, 445- 450. http://dx.doi.org/10.1515/IJNSNS.2007.8.3.445

[81] Marek-Crnjac, L. and He, J. (2013) International Journal of Astronomy and Astrophysics, 3, 464-471. http://dx.doi.org/10.4236/ijaa.2013.34053

[82] El Naschie, M.S. (1997) Chaos, Solitons \& Fractals, 8, 847-850. http://dx.doi.org/10.1016/S0960-0779(97)00084-2

[83] El Naschie, M.S. (2006) International Journal of Nonlinear Sciences and Numerical Simulation, 7, 97-100. http://dx.doi.org/10.1515/ijnsns.2006.7.1.97

[84] El Naschie, M.S. (2015) World Journal of Condensed Matter Physics, 5, 249. http://dx.doi.org/10.4236/wjcmp.2015.54026

[85] El Naschie, M.S. (2014) Journal of Modern Physics, 5, Article ID: 47094. http://dx.doi.org/10.4236/jmp.2014.59084

[86] El Naschie, M.S. (2005) Chaos, Solitons \& Fractals, 26, 257-261. http://dx.doi.org/10.1016/j.chaos.2004.12.024

[87] El Naschie, M.S. (2005) Chaos, Solitons \& Fractals, 25, 759-764. http://dx.doi.org/10.1016/j.chaos.2004.12.010

[88] El Naschie, M.S. (1998) Chaos, Solitons \& Fractals, 9, 913-919. http://dx.doi.org/10.1016/S0960-0779(97)00165-3

[89] El Naschie, M.S. (2013) Journal of Modern Physics and Applications, 2, 88-96.

[90] El Naschie, M.S. (2005) Chaos, Solitons \& Fractals, 24, 941-946. http://dx.doi.org/10.1016/j.chaos.2004.10.001

[91] El Naschie, M.S. (2014) International Journal of Astronomy and Astrophysics, 4, 332. http://dx.doi.org/10.4236/ijaa.2014.42027

[92] El Naschie, M.S. (2006) International Journal of Nonlinear Sciences and Numerical Simulation, 7, 119-128. http://dx.doi.org/10.1515/IJNSNS.2006.7.2.119

[93] El Naschie, M.S. (1998) International Journal of Theoretical Physics, 37, 2935-2951. http://dx.doi.org/10.1023/A:1026679628582

[94] El Naschie, M.S. (2013) Open Journal of Microphysics, 3, 64-70. http://dx.doi.org/10.4236/ojm.2013.33012

[95] El Naschie, M.S. (2008) Chaos, Solitons \& Fractals, 35, 320-322. http://dx.doi.org/10.1016/j.chaos.2007.06.110

[96] Iovane, G. (2006) International Journal of Nonlinear Sciences and Numerical Simulation, 7, 155-162. http://dx.doi.org/10.1515/IJNSNS.2006.7.2.155

[97] El Naschie, M.S. (2014) Open Journal of Philosophy, 4, 157-159. http://dx.doi.org/10.4236/ojpp.2014.42022

[98] El Naschie, M.S. (2007) Chaos, Solitons \& Fractals, 32, 427- 430. http://dx.doi.org/10.1016/j.chaos.2006.09.016

[99] El Naschie, M.S. (2008) Chaos, Solitons \& Fractals, 37, 16-22. http://dx.doi.org/10.1016/j.chaos.2007.09.079

[100] El Naschie, M.S. (2015) Open Journal of Applied Sciences, 5, 313. http://dx.doi.org/10.4236/ojapps.2015.57032

[101] He, J.H. (2007) International Journal of Nonlinear Sciences and Numerical Simulation, 8, 1-4. http://dx.doi.org/10.1515/IJNSNS.2007.8.1.1

[102] El Naschie, M.S. (2004) Chaos, Solitons \& Fractals, 19, 689-697. http://dx.doi.org/10.1016/S0960-0779(03)00337-0 
[103] El Naschie, M.S. (2014) Journal of Applied Mathematics and Physics, 2, 634-638. http://dx.doi.org/10.4236/jamp.2014.27069

[104] El Naschie, M.S. (2005) Chaos, Solitons \& Fractals, 23, 1091-1098. http://dx.doi.org/10.1016/j.chaos.2004.08.001

[105] He, J.H. (2006) Chaos, Solitons \& Fractals, 28, 285-289. http://dx.doi.org/10.1016/j.chaos.2005.08.001

[106] He, J.H. and Marek-Crnjac, L. (2013) International Journal of Modern Nonlinear Theory and Application, 2, Article ID:28827. http://dx.doi.org/10.4236/ijmnta.2013.21006

[107] El Naschie, M.S. (1998) Biosystems, 46, 41-46. http://dx.doi.org/10.1016/S0303-2647(97)00079-8

[108] El Naschie, M.S. (2005) Chaos, Solitons \& Fractals, 23, 1521-1525. http://dx.doi.org/10.1016/j.chaos.2004.09.003

[109] El Naschie, M.S. (2001) Chaos, Solitons \& Fractals, 12, 969-988. http://dx.doi.org/10.1016/S0960-0779(00)00263-0

[110] El Naschie, M.S. (2006) Chaos, Solitons \& Fractals, 30, 1025-1033. http://dx.doi.org/10.1016/j.chaos.2006.05.088

[111] El Naschie, M.S. (2013) Journal of Physics, 2, 18-23.

[112] El Naschie, M.S., Marek-Crnjac, L., Helal, M.A. and He, J.H. (2014) Applied Mathematics, 5, 1780-1790. http://dx.doi.org/10.4236/am.2014.512171

[113] Sigalotti, L.D.G. and Mejias, A. (2006) Chaos, Solitons \& Fractals, 30, 521-524. http://dx.doi.org/10.1016/j.chaos.2006.03.005

[114] Castro, C., El-Naschie, M.S. and Granik, A. (2000) Why We Live in 3+ 1 Dimensions. CERN Document Server. (No. hep-th/0004152).

[115] L Marek, C. and El Naschie, M.S. (2013) Journal of Modern Physics, 4, 31-38. http://dx.doi.org/10.4236/jmp.2013.411A005

[116] El Naschie, M.S. (2016) Journal of Quantum Information Science, 6, 1-9. http://dx.doi.org/10.4236/jqis.2016.61001

[117] El Naschie, M.S. (1993) Chaos, Solitons \& Fractals, 3, 89-98. http://dx.doi.org/10.1016/0960-0779(93)90042-Y

[118] El Naschie, M.S. (1995) Computers \& Mathematics with Applications, 29, 103-110. http://dx.doi.org/10.1016/0898-1221(95)00062-4

[119] El Naschie, M.S. (2003) Chaos, Solitons \& Fractals, 16, 637-649. http://dx.doi.org/10.1016/S0960-0779(02)00489-7

[120] El Naschie, M.S. (2014) Journal of Electromagnetic Analysis and Applications, 6, 233. http://dx.doi.org/10.4236/jemaa.2014.69023

[121] El Naschie, M.S. (2013) American Journal of Modern Physics, 2, 357-361. http://dx.doi.org/10.11648/j.ajmp.20130206.23

[122] El Naschie, M.S. (2015) Natural Science, 7, 210. http://dx.doi.org/10.4236/ns.2015.74024

[123] Castro, C. (2000) Chaos, Solitons \& Fractals, 11, 1663-1670. http://dx.doi.org/10.1016/S0960-0779(00)00018-7

[124] El Naschie, M.S. (2014) International Journal of Modern Nonlinear Theory and Application, 3, 1-5. http://dx.doi.org/10.4236/ijmnta.2014.31001

[125] El Naschie, M.S. (2004) International Journal of Modern Physics E, 13, 835-849. http://dx.doi.org/10.1142/S0218301304002429 
[126] El Naschie, M.S. (2000) Chaos, Solitons \& Fractals, 11, 1459-1469. http://dx.doi.org/10.1016/S0960-0779(99)00194-0

[127] El Naschie, M.S. (2014) Journal of Applied Mathematics and Physics, 2, 803. http://dx.doi.org/10.4236/jamp.2014.28088

[128] El Naschie, M.S. (2001) Chaos, Solitons \& Fractals, 12, 539-549. http://dx.doi.org/10.1016/S0960-0779(00)00187-9

[129] El Naschie, M.S. (2005) Chaos, Solitons \& Fractals, 23, 711-726. http://dx.doi.org/10.1016/j.chaos.2004.06.048

[130] El Naschie, M.S. (2015) Journal of Modern Physics, 6, 1321. http://dx.doi.org/10.4236/jmp.2015.69137

[131] Iovane, G. and Giordano, P. (2007) Chaos, Solitons \& Fractals, 32, 896-910. http://dx.doi.org/10.1016/j.chaos.2005.11.097

[132] He, J.H., Liu, Y., Xu, L. and Yu, J.Y. (2007) Chaos, Solitons \& Fractals, 32, 1096-1100. http://dx.doi.org/10.1016/j.chaos.2006.07.045

[133] Chen, W. (2006) Chaos, Solitons \& Fractals, 28, 923-929. http://dx.doi.org/10.1016/j.chaos.2005.08.199

[134] El Naschie, M.S. (2006) Chaos, Solitons \& Fractals, 29, 803-807. http://dx.doi.org/10.1016/j.chaos.2006.01.012

[135] El Naschie, M.S. (2008) Chaos, Solitons \& Fractals, 37, 662-668. http://dx.doi.org/10.1016/j.chaos.2008.01.018

[136] El Naschie, M.S. (2015) World Journal of Nano Science and Engineering, 5, 57. http://dx.doi.org/10.4236/wjnse.2015.53008

[137] El Naschie, M.S. (2005) Chaos, Solitons \& Fractals, 24, 899-905. http://dx.doi.org/10.1016/j.chaos.2004.11.003

[138] El Naschie, M.S. (2015) Open Journal of Philosophy, 5, 123. http://dx.doi.org/10.4236/ojpp.2015.51014

[139] El Naschie, M.S. (2014) Journal of Modern Physics and Applications, 2014, Article-ID: 6.

[140] Marek-Crnjac, L. and El Naschie, M.S. (2013) Applied Mathematics, 4, 22. http://dx.doi.org/10.4236/am.2013.411A2005

[141] Nottale, L. (1999) Chaos, Solitons \& Fractals, 10, 459-468. http://dx.doi.org/10.1016/S0960-0779(98)00195-7

[142] El Naschie, M.S. (2013) International Journal of Modern Nonlinear Theory and Application, 2, 167. http://dx.doi.org/10.4236/ijmnta.2013.23023

[143] El Naschie, M.S. (2005) Chaos, Solitons \& Fractals, 25, 935-939. http://dx.doi.org/10.1016/j.chaos.2005.02.029

[144] He, J.H. (2007) Chaos, Solitons \& Fractals, 32, 1645-1648. http://dx.doi.org/10.1016/j.chaos.2006.08.015

[145] Gottlieb, I., Agop, M., Ciobanu, G. and Stroe, A. (2006) Chaos, Solitons \& Fractals, 30, 380398. http://dx.doi.org/10.1016/j.chaos.2005.11.018

[146] El Naschie, M.S. (2015) The Cantorian Monadic Plasma behind the Zero Point Vacuum Spacetime Energy. American Journal of Nano Research and Application, 3, .

[147] Gottlieb, I., Agop, M. and Jarcău, M. (2004) Chaos, Solitons \& Fractals, 19, 705-730. http://dx.doi.org/10.1016/S0960-0779(03)00244-3

[148] El Naschie, M.S. (2009) Chaos, Solitons \& Fractals, 41, 2725-2732. http://dx.doi.org/10.1016/j.chaos.2008.10.001 
[149] El Naschie, M.S. (2008) Chaos, Solitons \& Fractals, 36, 1-17. http://dx.doi.org/10.1016/j.chaos.2007.08.058

[150] El Naschie, M.S. (2001) Chaos, Solitons \& Fractals, 12, 741-746. http://dx.doi.org/10.1016/S0960-0779(00)00193-4

[151] El Naschie, M.S. (2005) Chaos, Solitons \& Fractals, 26, 1-6. http://dx.doi.org/10.1016/j.chaos.2005.02.031

[152] El Naschie, M.S. and Rossler, O.E. (1994) Chaos, Solitons \& Fractals, 4, 307-309. http://dx.doi.org/10.1016/0960-0779(94)90049-3

[153] Nottale, L. (1995) Chaos, Solitons \& Fractals, 6, 399-410. http://dx.doi.org/10.1016/0960-0779(95)80047-K

[154] Marek-Crnjac, L. (2009) Chaos, Solitons \& Fractals, 41, 2697-2705. http://dx.doi.org/10.1016/j.chaos.2008.10.007

[155] Marek-Crnjac, L. (2015) Natural Science, 7, 581. http://dx.doi.org/10.4236/ns.2015.713058

[156] He, J.H. (2014) International Journal of Theoretical Physics, 53, 3698-3718. http://dx.doi.org/10.1007/s10773-014-2123-8

[157] El Naschie, M.S. (2005) Chaos, Solitons \& Fractals, 26, 665-670. http://dx.doi.org/10.1016/j.chaos.2005.01.018

[158] El Naschie, M.S. (2005) Chaos, Solitons \& Fractals, 23, 1511-1514. http://dx.doi.org/10.1016/j.chaos.2004.08.008

[159] Agop, M., Griga, V., Ciobanu, B., Ciubotariu, C., Buzea, C.G., Stan, C. and Buzea, C. (1998) Chaos, Solitons \& Fractals, 9, 1143-1181. http://dx.doi.org/10.1016/S0960-0779(98)80005-2

[160] Giordano, P., Iovane, G. and Laserra, E. (2007) Chaos, Solitons \& Fractals, 31, 1108-1117. http://dx.doi.org/10.1016/j.chaos.2006.03.114

[161] El Naschie, M.S. (2005) Chaos, Solitons \& Fractals, 24, 29-32. http://dx.doi.org/10.1016/j.chaos.2004.09.002

[162] He, J.H. (2007) Chaos, Solitons \& Fractals, 31, 782- 786. http://dx.doi.org/10.1016/j.chaos.2006.04.041

[163] Iovane, G., Giordano, P. and Salerno, S. (2005) Chaos, Solitons \& Fractals, 24, 423-441. http://dx.doi.org/10.1016/j.chaos.2004.09.068

[164] El Naschie, M.S. (2003) On John Nash's Crumpled Surface. Chaos, Solitons \& Fractals, 18, 635-641. http://dx.doi.org/10.1016/S0960-0779(03)00007-9

[165] El Naschie, M.S. (2016) International Journal of Astronomy and Astrophysics, 6, 135. http://dx.doi.org/10.4236/ijaa.2016.62011

[166] El Naschie, M.S. (2008) Chaos, Solitons \& Fractals, 35, 268-273. http://dx.doi.org/10.1016/j.chaos.2007.07.036

[167] El Naschie, M.S. (2015) World Journal of Nano Science and Engineering, 5, 26. http://dx.doi.org/10.4236/wjnse.2015.51004

[168] El Naschie, M.S. (2008) Chaos, Solitons \& Fractals, 37, 346-359. http://dx.doi.org/10.1016/j.chaos.2007.10.021

[169] El Naschie, M.S. (2015) Open Journal of Philosophy, 5, 319. http://dx.doi.org/10.4236/ojpp.2015.56040

[170] El Naschie, M.S. (2015) International Journal of Astronomy and Astrophysics, 5, 243. http://dx.doi.org/10.4236/ijaa.2015.54027

[171] Rössler, O.E. (1996) Chaos, Solitons \& Fractals, 7, 845-852. http://dx.doi.org/10.1016/0960-0779(95)00117-4 
[172] Nottale, L. (1998) Chaos, Solitons \& Fractals, 9, 1051-1061. http://dx.doi.org/10.1016/S0960-0779(97)00190-2

[173] El Naschie, M.S. (2005) Chaos, Solitons \& Fractals, 25, 531-533. http://dx.doi.org/10.1016/j.chaos.2005.01.001

[174] Iovane, G. (2006) Chaos, Solitons \& Fractals, 29, 1-22. http://dx.doi.org/10.1016/j.chaos.2005.10.045

[175] Czajko, J. (2000) Chaos, Solitons \& Fractals, 11, 1983-1992. http://dx.doi.org/10.1016/S0960-0779(99)00091-0

[176] El Naschie, M.S. (2005) Chaos, Solitons \& Fractals, 26, 673-676. http://dx.doi.org/10.1016/j.chaos.2005.02.030

[177] Nottale, L. (1994) Chaos, Solitons \& Fractals, 4, 361-388. http://dx.doi.org/10.1016/0960-0779(94)90051-5

[178] El Naschie, M.S. (2015) Open Journal of Microphysics, 5, 11. http://dx.doi.org/10.4236/ojm.2015.52002

[179] El Naschie, M.S. (2004) Quantum Collapse of Wave Interference Pattern in the Two-Slit Experiment: A Set Theoretical Resolution. In Other Words, 500(1/2).

[180] Iovane, G. (2006) Chaos, Solitons \& Fractals, 28, 857-878. http://dx.doi.org/10.1016/j.chaos.2005.08.074

[181] El Naschie, M.S. (1992) Chaos, Solitons \& Fractals, 2, 91-94. http://dx.doi.org/10.1016/0960-0779(92)90050-W

[182] Iovane, G., Gargiulo, G. and Zappale, E. (2006) Chaos, Solitons \& Fractals, 27, 588-598. http://dx.doi.org/10.1016/j.chaos.2005.05.015

[183] El Naschie, M.S. (1998) Chaos, Solitons \& Fractals, 9, 1445-1471. http://dx.doi.org/10.1016/S0960-0779(98)00120-9

[184] El Naschie, M.S. (2006) Chaos, Solitons \& Fractals, 28, 1366-1371. http://dx.doi.org/10.1016/j.chaos.2005.11.001

[185] El Naschie, M.S. (2015) Journal of Modern Physics, 6, 384. http://dx.doi.org/10.4236/jmp.2015.64042

[186] Elnaschie, M.S. (2005) International Journal of Nonlinear Sciences and Numerical Simulation, 6, 335-342. http://dx.doi.org/10.1515/IJNSNS.2005.6.4.335

[187] Iovane, G., Chinnici, M. and Tortoriello, F.S. (2008) Chaos, Solitons \& Fractals, 35, 645658. http://dx.doi.org/10.1016/j.chaos.2007.07.051

[188] El Naschie, M.S. (2015) World Journal of Nano Science and Engineering, 5, 49. http://dx.doi.org/10.4236/wjnse.2015.52007

[189] El Naschie, M.S. (2014) Advances in Pure Mathematics, 4, 641. http://dx.doi.org/10.4236/apm.2014.412073

[190] Selvam, A.M. and Fadnavis, S. (1999) Chaos, Solitons \& Fractals, 10, 1321-1334. http://dx.doi.org/10.1016/S0960-0779(98)00150-7

[191] El Naschie, M.S. (2006) Chaos, Solitons \& Fractals, 30, 636-641. http://dx.doi.org/10.1016/j.chaos.2006.04.044

[192] He, J.H. (2006) Chaos, Solitons \& Fractals, 30, 506-511. http://dx.doi.org/10.1016/j.chaos.2005.11.033

[193] Marek-Crnjac, L. (2013) American Journal of Modern Physics, 2, 255-263. http://dx.doi.org/10.11648/j.ajmp.20130205.14

[194] El-Ahmady, A.E. and Rafat, H. (2006) Chaos, Solitons \& Fractals, 30, 836-844. http://dx.doi.org/10.1016/j.chaos.2005.05.033 
[195] El Naschie, M.S. (2016) Natural Science, 8, 152. http://dx.doi.org/10.4236/ns.2016.83018

[196] Selvam, A.M. and Fadnavis, S. (1999) Chaos, Solitons \& Fractals, 10, 1577-1582. http://dx.doi.org/10.1016/S0960-0779(98)00209-4

[197] He, J.H. and Marek-Crnjac, L. (2013) Fractal Spacetime and Noncommutative Geometry in Quantum and High Energy Physics, 3, 130-137.

[198] El Naschie, M.S. (2008) Chaos, Solitons \& Fractals, 35, 99-103. http://dx.doi.org/10.1016/j.chaos.2007.05.005

[199] El Naschie, M.S. (2007) International Journal of Nonlinear Sciences and Numerical Simulation, 8, 195-198. http://dx.doi.org/10.1515/ijnsns.2007.8.2.195

[200] El Naschie, M.S. (2009) Chaos, Solitons \& Fractals, 41, 869-874. http://dx.doi.org/10.1016/j.chaos.2008.04.013

[201] El Naschie, M.S. (1994) Chaos, Solitons \& Fractals, 4, 1235-1247. http://dx.doi.org/10.1016/0960-0779(94)90034-5

[202] Özgür, C. (2008) Chaos, Solitons \& Fractals, 38, 1373-1377. http://dx.doi.org/10.1016/j.chaos.2008.03.016

[203] He, J.H. (2007) Chaos, Solitons \& Fractals, 34, 727-729. http://dx.doi.org/10.1016/j.chaos.2006.04.052

[204] Castro, C. (2001) Chaos, Solitons \& Fractals, 12, 101-104. http://dx.doi.org/10.1016/S0960-0779(00)00196-X

[205] Rami, E.N.A. (2009) Chaos, Solitons \& Fractals, 42, 84-88. http://dx.doi.org/10.1016/j.chaos.2008.10.031

[206] Babchin, A.J. and El Naschie, M.S. (2015) World Journal of Condensed Matter Physics, 6, 1. http://dx.doi.org/10.4236/wjcmp.2016.61001

[207] He, J.H. (2008) Chaos, Solitons \& Fractals, 36, 542-545. http://dx.doi.org/10.1016/j.chaos.2007.07.093

[208] Nagasawa, M. (1996) Chaos, Solitons \& Fractals, 7, 631-643. http://dx.doi.org/10.1016/0960-0779(95)00115-8

[209] Iovane, G., Giordano, P. and Laserra, E. (2004) Chaos, Solitons \& Fractals, 22, 975-983. http://dx.doi.org/10.1016/j.chaos.2004.04.019

[210] El Naschie, M.S. (2015) Natural Science, 7, 287. http://dx.doi.org/10.4236/ns.2015.76032

[211] Ord, G.N. (1997) Chaos, Solitons \& Fractals, 8, 727-741. http://dx.doi.org/10.1016/S0960-0779(96)00059-8

[212] Agop, M., Paun, V. and Harabagiu, A. (2008) Chaos, Solitons \& Fractals, 37, 1269-1278. http://dx.doi.org/10.1016/j.chaos.2008.01.006

[213] El Naschie, M.S., Marek-Crnjac, L., He, J.H. and Helal, M.A. (2013) Fractal Spacetime and Noncommutative Geometry in Quantum and High Energy Physics, 3, 3-10.

[214] El Naschie, M.S. (2005) Chaos, Solitons \& Fractals, 26, 247-254. http://dx.doi.org/10.1016/j.chaos.2005.01.016

[215] Nottale, L. (2005) Chaos, Solitons \& Fractals, 25, 797-803. http://dx.doi.org/10.1016/j.chaos.2004.11.071

[216] El Naschie, M.S. (2007) Chaos, Solitons \& Fractals, 32, 893-895. http://dx.doi.org/10.1016/j.chaos.2006.09.055

[217] Saniga, M. (2001) Chaos, Solitons \& Fractals, 12, 2127-2142. http://dx.doi.org/10.1016/S0960-0779(00)00183-1 
[218] El Naschie, M.S. (1995) Quantum Measurement, Information, Diffusion and Cantorian Geodesies. Quantum Mechanics, Diffusion and Chaotic Fractals, Pergamon Press, Oxford.

[219] Mejias, A., Sigalotti, L.D.G., Sira, E. and De Felice, F. (2004) Chaos, Solitons \& Fractals, 19, 773-777. http://dx.doi.org/10.1016/S0960-0779(03)00273-X

[220] Munceleanu, G.V., Paun, V.P., Casian-Botez, I. and Agop, M. (2011) International Journal of Bifurcation and Chaos, 21, 603-618. http://dx.doi.org/10.1142/S021812741102888X

[221] Ho, M.E.N. and Giuseppe Vitiello, M.W. (2015) Is Spacetime Fractal and Quantum Coherent in the Golden Mean? Global Journal of Science Frontier Research, 15.

[222] El Naschie, M.S. (2003) Chaos, Solitons \& Fractals, 17, 989-1001. http://dx.doi.org/10.1016/S0960-0779(03)00006-7

[223] El Naschie, M.S. (2006) International Journal of Nonlinear Sciences and Numerical Simulation, 7, 1-6. http://dx.doi.org/10.1515/IJNSNS.2006.7.1.1

[224] Castro, C., Granik, A. and El Naschie, M.S. (2000) Why We Live in 3 Dimensions. arXiv preprint hep-th/0004152.

[225] Selvam, A.M. (2005) A General Systems Theory for Chaos, Quantum Mechanics and Gravity for Dynamical Systems of All Space-Time Scales. arXiv preprint physics/0503028.

[226] Iovane, G. and Benedetto, E. (2006) Chaos, Solitons \& Fractals, 30, 269-277. http://dx.doi.org/10.1016/j.chaos.2005.11.005

[227] Goldfain, E. (2005) Chaos, Solitons \& Fractals, 23, 701-710. http://dx.doi.org/10.1016/j.chaos.2004.05.020

[228] El Naschie, M.S. (2016) Journal of Modern Physics, 7, 729. http://dx.doi.org/10.4236/jmp.2016.78069

[229] El Naschie, M.S. (2007) International Journal of Nonlinear Sciences and Numerical Simulation, 8, 5-10. http://dx.doi.org/10.1515/IJNSNS.2007.8.1.5

[230] El Naschie, M.S. (2009) Chaos, Solitons \& Fractals, 41, 2787-2789. http://dx.doi.org/10.1016/j.chaos.2008.10.011

[231] El Naschie, M.S. (2016) World Journal of Condensed Matter Physics, 6, 63. http://dx.doi.org/10.4236/wjcmp.2016.62009

[232] El Naschie, M.S. (2015) American Journal of Nano Research and Applications, 3, 33-40.

[233] El Naschie, M.S. (2000) Chaos, Solitons \& Fractals, 11, 2391-2395. http://dx.doi.org/10.1016/S0960-0779(99)00209-X

[234] Stakhov, A. and Rozin, B. (2005) Chaos, Solitons \& Fractals, 26, 677-684. http://dx.doi.org/10.1016/j.chaos.2005.01.057

[235] He, J.H. (2009) Chaos, Solitons \& Fractals, 42, 2754-2759. http://dx.doi.org/10.1016/j.chaos.2009.03.182

[236] El Naschie, M.S. (2016) International Journal of Astronomy and Astrophysics, 6, 56. http://dx.doi.org/10.4236/ijaa.2016.61005

[237] El Naschie, M.S. (2005) Chaos, Solitons \& Fractals, 25, 545-548. http://dx.doi.org/10.1016/j.chaos.2005.01.009

[238] Sidharth, B.G. (2003) Chaos, Solitons \& Fractals, 18, 197-201. http://dx.doi.org/10.1016/S0960-0779(02)00632-X

[239] El Naschie, M.S. (2004) Chaos, Solitons \& Fractals, 22, 1199-1209. http://dx.doi.org/10.1016/j.chaos.2004.04.026

[240] El Naschie, M.S. (1999) Chaos, Solitons \& Fractals, 10, 1955-1965. http://dx.doi.org/10.1016/S0960-0779(99)00030-2 
[241] Dariescu, M.A., Dariescu, C. and Pîrghie, A.C. (2009) Chaos, Solitons \& Fractals, 42, 247252. http://dx.doi.org/10.1016/j.chaos.2008.11.021

[242] Maker, D. (1999) Chaos, Solitons \& Fractals, 10, 31-42. http://dx.doi.org/10.1016/S0960-0779(98)00108-8

[243] El Naschie, M.S. (2008) Chaos, Solitons \& Fractals, 38, 1051-1053. http://dx.doi.org/10.1016/j.chaos.2008.06.001

[244] Iovane, G., Laserra, E. and Giordano, P. (2004) Chaos, Solitons \& Fractals, 22, 521-528. http://dx.doi.org/10.1016/j.chaos.2004.02.026

[245] He, J.H. and Xu, L. (2009) Chaos, Solitons \& Fractals, 39, 2119-2124. http://dx.doi.org/10.1016/j.chaos.2007.06.088

[246] Naschie, M.E. (2006) International Journal of Nonlinear Sciences and Numerical Simulation, 7, 477-482. http://dx.doi.org/10.1515/IJNSNS.2006.7.4.477

[247] Auffray, J.P. (2014) Journal of Modern Physics, 5, 1427. http://dx.doi.org/10.4236/jmp.2014.515144

[248] El Naschie, M.S. (2015) Journal of Quantum Information Science, 5, 24. http://dx.doi.org/10.4236/jqis.2015.51004

[249] El Naschie, M.S. (2006) Chaos, Solitons \& Fractals, 29, 871-875. http://dx.doi.org/10.1016/j.chaos.2006.01.005

[250] El Naschie, M.S. (2016) Journal of Quantum Information Science, 6, 57. http://dx.doi.org/10.4236/jqis.2016.62007

[251] El-Ahmady, A.E. and Al-Hesiny, E. (2011) The International Journal of Nonlinear Science, 11, 451-458.

[252] Iovane, G. (2004) Chaos, Solitons \& Fractals, 20, 657-667. http://dx.doi.org/10.1016/j.chaos.2003.09.036

[253] El Naschie, M.S. (2005) Chaos, Solitons \& Fractals, 26, 303-311. http://dx.doi.org/10.1016/j.chaos.2005.03.004

[254] El Naschie, M.S. (2015) Natural Science, 7, 483. http://dx.doi.org/10.4236/ns.2015.710049

[255] Marek-Crnjac, L. (2003) Chaos, Solitons \& Fractals, 18, 125-133. http://dx.doi.org/10.1016/S0960-0779(02)00587-8

[256] Marek-Crnjac, L. (2009) Chaos, Solitons \& Fractals, 42, 1796-1799. http://dx.doi.org/10.1016/j.chaos.2009.03.094

[257] Özgür, C. (2009) Chaos, Solitons \& Fractals, 40, 1156-1161. http://dx.doi.org/10.1016/j.chaos.2007.08.070

[258] Sidharth, B.G. (2002) Chaos, Solitons \& Fractals, 13, 189-193. http://dx.doi.org/10.1016/S0960-0779(00)00269-1

[259] Iovane, G. (2006) Chaos, Solitons \& Fractals, 27, 618-629. http://dx.doi.org/10.1016/j.chaos.2005.04.093

[260] El Naschie, M.S. (2008) Chaos, Solitons \& Fractals, 35, 664-667. http://dx.doi.org/10.1016/j.chaos.2007.07.082

[261] Tanaka, Y., Mizuno, Y. and Kado, T. (2005) Chaos, Solitons \& Fractals, 24, 407-422. http://dx.doi.org/10.1016/j.chaos.2004.09.034

[262] Auffray, J.P. (2015) Journal of Modern Physics, 6, 536. http://dx.doi.org/10.4236/jmp.2015.65058

[263] Martienssen, W. (2005) Chaos, Solitons \& Fractals, 25, 805-806. http://dx.doi.org/10.1016/j.chaos.2005.02.001 
[264] Agop, M. and Vasilica, M. (2006) Chaos, Solitons \& Fractals, 30, 318-323. http://dx.doi.org/10.1016/j.chaos.2006.01.105

[265] Chen, Q. and Shi, Z. (2008) Chaos, Solitons \& Fractals, 35, 323-332. http://dx.doi.org/10.1016/j.chaos.2007.06.065

[266] Qiu, H. and Su, W. (2007) Chaos, Solitons \& Fractals, 33, 1625-1634. http://dx.doi.org/10.1016/j.chaos.2006.03.024

[267] Nottale, L. (2001) Revue de synthèse, 122, 11-25. http://dx.doi.org/10.1007/BF02990499

[268] El Naschie, M.S. (2003) Chaos, Solitons \& Fractals, 17, 631-638. http://dx.doi.org/10.1016/S0960-0779(02)00630-6

[269] Vrobel, S. (2011) Why a Watched Kettle Never Boils. World Scientific, Singapore.

[270] Gottlieb, I., Ciobanu, G. and Buzea, C.G. (2003) Chaos, Solitons \& Fractals, 17, 789-796. http://dx.doi.org/10.1016/S0960-0779(02)00484-8

[271] He, J.H. (2009) Chaos, Solitons \& Fractals, 41, 2533-2537. http://dx.doi.org/10.1016/j.chaos.2008.09.027

[272] Argyris, J., Ciubotariu, C.I. and Weingaertner, W.E. (2000) Chaos, Solitons \& Fractals, 11, 1671-1719. http://dx.doi.org/10.1016/S0960-0779(99)00065-X

[273] El Naschie, M.S. (2007) Chaos, Solitons \& Fractals, 34, 1377-1381. http://dx.doi.org/10.1016/j.chaos.2007.02.016

[274] Agop, M. and Craciun, P. (2006) Chaos, Solitons \& Fractals, 30, 30-40. http://dx.doi.org/10.1016/j.chaos.2006.01.006

[275] Agop, M., Ioannou, P.D. and Buzea, C.G. (2002) Chaos, Solitons \& Fractals, 13, 1137-1165. http://dx.doi.org/10.1016/S0960-0779(01)00123-0

[276] Sidharth, B.G. (2001) A Reconciliation of Electromagnetism and Gravitation. arXiv preprint physics/0110040.

[277] El-Nabulsi, A.R. (2009) Chaos, Solitons \& Fractals, 42, 2924-2933. http://dx.doi.org/10.1016/j.chaos.2009.04.004

[278] Weiss, H. and Weiss, V. (2003) Chaos, Solitons \& Fractals, 18, 643-652. http://dx.doi.org/10.1016/S0960-0779(03)00026-2

[279] Wu, G.C. and He, J.H. (2009) Chaos, Solitons \& Fractals, 42, 781-783. http://dx.doi.org/10.1016/j.chaos.2009.02.007

[280] Czajko, J. (2004) Chaos, Solitons \& Fractals, 21, 261-271. http://dx.doi.org/10.1016/j.chaos.2003.12.046

[281] Sidharth, B.G. (2002) Chaos, Solitons \& Fractals, 13, 617-620. http://dx.doi.org/10.1016/S0960-0779(01)00017-0

[282] De, A., De, U.C. and Gazi, A.K. (2011) Communications of the Korean Mathematical Society, 26, 623-634. http://dx.doi.org/10.4134/CKMS.2011.26.4.623

[283] El Naschie, M.S. (2008) Chaos, Solitons \& Fractals, 36, 521-525. http://dx.doi.org/10.1016/j.chaos.2007.09.004

[284] Sidharth, B.G. (2000) Chaos, Solitons \& Fractals, 11, 1045-1046. http://dx.doi.org/10.1016/S0960-0779(98)00331-2

[285] El Naschie, M. S. (2001) Chaos, Solitons \& Fractals, 12, 1361-1368. http://dx.doi.org/10.1016/S0960-0779(01)00008-X

[286] Iovane, G., Laserra, E. and Tortoriello, F.S. (2004) Chaos, Solitons \& Fractals, 20, 415-426. http://dx.doi.org/10.1016/j.chaos.2003.08.004 
[287] Saniga, M. (2002) Chaos, Solitons \& Fractals, 13, 807-814. http://dx.doi.org/10.1016/S0960-0779(01)00056-X

[288] Nottale, L. (2001) Chaos, Solitons \& Fractals, 12, 1577-1583. http://dx.doi.org/10.1016/S0960-0779(01)00015-7

[289] El Naschie, M.S. (2008) Chaos, Solitons \& Fractals, 37, 6-8. http://dx.doi.org/10.1016/j.chaos.2007.09.057

[290] El Naschie, M.S., Olsen, S. and He, J.H. (2013) Fractal Spacetime and Noncommutative Geometry, 3, 11-20.

[291] El Naschie, M.S. (1997) Chaos, Solitons \& Fractals, 8, vii-x. http://dx.doi.org/10.1016/S0960-0779(97)88695-X

[292] El Naschie, M.S. (2004) Chaos, Solitons \& Fractals, 20, 649-654. http://dx.doi.org/10.1016/j.chaos.2003.10.010

[293] Xu, L. and Zhong, T. (2011) Nonlinear Science Letters B, 1, 10-11.

[294] Giordano, P. (2006) International Journal of Nonlinear Sciences and Numerical Simulation, 7, 451-460. http://dx.doi.org/10.1515/IJNSNS.2006.7.4.451

[295] Nozari, K. and Mehdipour, S.H. (2009) Chaos, Solitons \& Fractals, 39, 956-970. http://dx.doi.org/10.1016/j.chaos.2007.02.018

[296] Benedetto, E. (2009) International Journal of Theoretical Physics, 48, 1603-1621. http://dx.doi.org/10.1007/s10773-009-9933-0

[297] El Naschie, M.S. (2007) Chaos, Solitons \& Fractals, 31, 521-526. http://dx.doi.org/10.1016/j.chaos.2006.06.028

[298] Zmeskal, O., Nezadal, M. and Buchnicek, M. (2003) Chaos, Solitons \& Fractals, 17, $113-$ 119. http://dx.doi.org/10.1016/S0960-0779(02)00412-5

[299] Castro, C. (2000) On the Four Dimensional Conformal Anomaly, Fractal Spacetime and the Fine Structure Constant. arXiv preprint physics/0010072.

[300] Marek-Crnjac, L. (2008) Chaos, Solitons \& Fractals, 37, 1279-1288. http://dx.doi.org/10.1016/j.chaos.2008.01.021

[301] Özgür, C. (2009) Chaos, Solitons \& Fractals, 39, 2457-2464. http://dx.doi.org/10.1016/j.chaos.2007.07.018

[302] Sidharth, B.G. (2001) Chaos, Solitons \& Fractals, 12, 2143-2147. http://dx.doi.org/10.1016/S0960-0779(00)00181-8

[303] Fred, Y.Y. (2009) From Chaos to Unification: U Theory vs. M Theory. Chaos, Solitons \& Fractals, 42, 89-93. http://dx.doi.org/10.1016/j.chaos.2008.10.030

[304] Colotin, M., Pompilian, G.O., Nica, P., Gurlui, S., Paun, V. and Agop, M. (2009) Acta Physica Polonica-Series A General Physics, 116, 157. http://dx.doi.org/10.12693/APhysPolA.116.157

[305] Rossler, O. E., Fröhlich, D., Movassagh, R. and Moore, A. (2007) Chaos, Solitons \& Fractals, 33, 770-775. http://dx.doi.org/10.1016/j.chaos.2006.06.046

[306] El Naschie, M.S. (2004) Chaos, Solitons \& Fractals, 20, 455-458. http://dx.doi.org/10.1016/j.chaos.2003.10.008

[307] Agop, M., Ioannou, P.D., Nica, P., Buzea, C.G. and Jarcau, M. (2003) Chaos, Solitons \& Fractals, 18, 1-16. http://dx.doi.org/10.1016/S0960-0779(02)00633-1

[308] Agop, M., Ciobanu, G. and Zaharia, L. (2003) Chaos, Solitons \& Fractals, 15, 445-453. http://dx.doi.org/10.1016/S0960-0779(02)00139-X 
[309] Rami, E.N.A. (2009) Chaos, Solitons \& Fractals, 41, 2262-2270. http://dx.doi.org/10.1016/j.chaos.2008.08.033

[310] Zmeskal, O., Vala, M., Weiter, M. and Stefkova, P. (2009) Chaos, Solitons \& Fractals, 42, 1878-1892. http://dx.doi.org/10.1016/j.chaos.2009.03.106

[311] Iovane, G., Bellucci, S. and Benedetto, E. (2008) Chaos, Solitons \& Fractals, 37, 49-59. http://dx.doi.org/10.1016/j.chaos.2007.09.022

[312] Goldfain, E. (2005) International Journal of Nonlinear Sciences and Numerical Simulation, 6, 351-356. http://dx.doi.org/10.1515/IJNSNS.2005.6.4.351

[313] de Felice, F., Sigalotti, L.D.G. and Mejias, A. (2004) Chaos, Solitons \& Fractals, 21, 573-578. http://dx.doi.org/10.1016/j.chaos.2003.12.091

[314] El Naschie, M.S. (2005) Chaos, Solitons \& Fractals, 23, 1941-1943. http://dx.doi.org/10.1016/j.chaos.2004.08.005

[315] Elokaby, A. (2009) Chaos, Solitons \& Fractals, 41, 1616-1618. http://dx.doi.org/10.1016/j.chaos.2008.07.003

[316] Nottale, L. (2000) Scale Relativity, Fractal Space-Time and Morphogenesis of Structures. H. In: Diebner, T.D. and Weibel, P., Eds., Sciences of the Interface, Genista, Tubingen, 38.

[317] Agop, M., Ioannou, P.D., Buzea, C. and Nica, P. (2003) Chaos, Solitons \& Fractals, 16, 321338. http://dx.doi.org/10.1016/S0960-0779(02)00413-7

[318] Rami, E.N.A. (2009) Chaos, Solitons \& Fractals, 42, 377-384. http://dx.doi.org/10.1016/j.chaos.2008.12.008

[319] El Naschie, M.S. (2000) Chaos, Solitons \& Fractals, 11, 2397-2408. http://dx.doi.org/10.1016/S0960-0779(00)00108-9

[320] El Naschie, M.S. and He, J.H. (2013) Fractal Spacetime and Non-Commutative Geometry in Quantum and High Energy Physics, 3, 106-119.

[321] Dickau, J.J. (2009) Fractal Cosmology. Chaos, Solitons \& Fractals, 41, 2103-2105. http://dx.doi.org/10.1016/j.chaos.2008.07.056

[322] El Naschie, M.S. (2008) Chaos, Solitons \& Fractals, 35, 684-687. http://dx.doi.org/10.1016/j.chaos.2007.07.084

[323] Tomaschitz, R. (1997) International Journal of Bifurcation and Chaos, 7, 1847-1853. http://dx.doi.org/10.1142/S0218127497001412

[324] Ho, M.W. (2014) Journal of the Institute of Science in Society, 62, 40-43.

[325] Sidharth, B.G. (2003) Chaos, Solitons \& Fractals, 15, 593-595. http://dx.doi.org/10.1016/S0960-0779(02)00159-5

[326] Ćirić, L.B., Ješić, S.N. and Ume, J.S. (2008) Chaos, Solitons \& Fractals, 37, 781-791. http://dx.doi.org/10.1016/j.chaos.2006.09.093

[327] El Naschie, M.S. (2005) Chaos, Solitons \& Fractals, 24, 659- 663. http://dx.doi.org/10.1016/j.chaos.2004.11.002

[328] Yildiz, A., De, U.C. and Cetinkaya, A. (2011) N (k)-Quasi Einstein Manifolds Satisfying Certain Curvature Conditions. The Dumlupnar University Research Foundation (No: 2011-25).

[329] Sidharth, B.G. (2002) Chaos, Solitons \& Fractals, 13, 1369-1370. http://dx.doi.org/10.1016/S0960-0779(01)00114-X

[330] Tanaka, Y. (2005) Chaos, Solitons \& Fractals, 23, 33-41. http://dx.doi.org/10.1016/j.chaos.2004.03.031

[331] Nagasawa, M. (1997) Chaos, Solitons \& Fractals, 8, 1773-1792. 
http://dx.doi.org/10.1016/S0960-0779(97)00036-2

[332] El Naschie, M.S. (2008) Chaos, Solitons \& Fractals, 35, 7-12. http://dx.doi.org/10.1016/j.chaos.2007.06.023

[333] El Naschie, M.S. (2008) Chaos, Solitons \& Fractals, 37, 1289-1291. http://dx.doi.org/10.1016/j.chaos.2008.02.002

[334] El Naschie, M.S. (2009) Chaos, Solitons \& Fractals, 41, 1340-1343. http://dx.doi.org/10.1016/j.chaos.2008.05.015

[335] Iovane, G. and Salerno, S. (2005) WSEAS Transactions on Mathematics, 4, 184.

[336] Liu, S.D., Liu, S.K., Fu, Z.T., Ren, K. and Guo, Y. (2003) Chaos, Solitons \& Fractals, 15, 627-630. http://dx.doi.org/10.1016/S0960-0779(02)00138-8

[337] Yang, C.D. (2008) Chaos, Solitons \& Fractals, 38, 316-331. http://dx.doi.org/10.1016/j.chaos.2008.01.019

[338] El Naschie, M.S., Olsen, S., He, J.H., Nada, S., Marek-Crnjac, L. and Helal, A. (2012) International Journal of Modern Nonlinear Theory and Application, 1, 84-92. http://dx.doi.org/10.4236/IJMNTA.2012.13012

[339] Marek-Crnjac, L. (2006) Chaos, Solitons \& Fractals, 27, 575-579. http://dx.doi.org/10.1016/j.chaos.2005.04.099

[340] Pavlos, G.P., Iliopoulos, A.C., Karakatsanis, L.P., Tsoutsouras, V.G. and Pavlos, E.G. (2011) Complexity Theory and Physical Unification: From Microscopic to Macroscopic Level. Chaos Theory: Modeling, Simulation and Applications, World Scientific Publishing, 297308. http://dx.doi.org/10.1142/9789814350341 0035

[341] El Naschie, M.S. (1993) Chaos, Solitons \& Fractals, 3, 481-488. http://dx.doi.org/10.1016/0960-0779(93)90032-V

[342] Gottlieb, I. and Agop, M. (2007) Chaos, Solitons \& Fractals, 34, 1025-1029. http://dx.doi.org/10.1016/j.chaos.2006.03.108

[343] El Naschie, M.S. (2013) Electromagnetic and Gravitational Origin of Dark Energy in KaluzaKlein D = 5 Spacetime. PIERS Proceeding, Stockholm, 91-97.

[344] Sigalotti, L.D.G. and Rendón, O. (2007) Chaos, Solitons \& Fractals, 32, 1611-1614. http://dx.doi.org/10.1016/j.chaos.2006.08.034

[345] Ord, G.N. (1996) Journal of Physics A: Mathematical and General, 29, L123. http://dx.doi.org/10.1088/0305-4470/29/5/007

[346] Saniga, M. (2005) Chaos, Solitons \& Fractals, 23, 645-650. http://dx.doi.org/10.1016/j.chaos.2004.05.018

[347] El Naschie, M.S. (2016) American Journal of Computational Mathematics, 6, 185. http://dx.doi.org/10.4236/ajcm.2016.63020

[348] El Naschie, M.S. (1996) Chaos, Solitons \& Fractals, 7, 1501-1506. http://dx.doi.org/10.1016/0960-0779(96)80001-B

[349] Rossler, O.E. and Kuypers, H. (2005) Chaos, Solitons \& Fractals, 25, 897-899. http://dx.doi.org/10.1016/j.chaos.2004.11.097

[350] El Naschie, M.S. (2016) Advances in Pure Mathematics, 6, 446. http://dx.doi.org/10.4236/apm.2016.66032

[351] El Naschie, M.S. (2013) Fractal Spacetime and Noncommutative Geometry in Quantum and High Energy Physics, 3, 35-38.

[352] El Naschie, M.S. (2008) Chaos, Solitons \& Fractals, 38, 609-611. http://dx.doi.org/10.1016/j.chaos.2008.04.015 
[353] He, J.H., Zhong, T., Xu, L., Marek-Crnjac, L., Nada, S.I. and Helal, M.A. (2011) Nonlinear Science Letters $B, 1,15-24$.

[354] El Naschie, M.S. (2008) Chaos, Solitons \& Fractals, 35, 303-307. http://dx.doi.org/10.1016/j.chaos.2007.07.025

[355] Agop, M. and Enache, V. (2007) Chaos, Solitons \& Fractals, 32, 296-301. http://dx.doi.org/10.1016/j.chaos.2006.04.068

[356] Ho, M.W. (2014) Golden Geometry of E-Infinity Fractal Spacetime. The Story of Phi, Part, 5.

[357] Agop, M. and Craciun, P. (2006) Chaos, Solitons \& Fractals, 30, 441-452. http://dx.doi.org/10.1016/j.chaos.2005.12.048

[358] El Naschie, M.S. (2008) Chaos, Solitons \& Fractals, 36, 1200-1204. http://dx.doi.org/10.1016/j.chaos.2007.09.039

[359] Marek-Crnjac, L. (2012) Quantum Gravity in Cantorian Space-Time. INTECH Open Access Publisher. http://dx.doi.org/10.5772/37232

[360] El Naschie, M.S. (2003) Chaos, Solitons \& Fractals, 17, 797-807. http://dx.doi.org/10.1016/S0960-0779(02)00684-7

[361] El Naschie, M.S. (2014) Natural Science, 6, 1259. http://dx.doi.org/10.4236/ns.2014.616115

[362] Agop, M. and Gottlieb, I. (2006) Journal of Mathematical Physics, 47, 53503-53503. http://dx.doi.org/10.1063/1.2196747

[363] Giné, J. (2008) Chaos, Solitons \& Fractals, 35, 1-6. http://dx.doi.org/10.1016/j.chaos.2007.06.097

[364] Auffray, J.P. (2015) Journal of Modern Physics, 6, 1478. http://dx.doi.org/10.4236/jmp.2015.611152

[365] Agop, M., Ioannou, P.D., Nica, P., Găluşcă, G. and Ştefan, M. (2005) Chaos, Solitons \& Fractals, 23, 1497-1509. http://dx.doi.org/10.1016/S0960-0779(04)00439-4

[366] He, J.H. and Huang, Z. (2006) Chaos, Solitons \& Fractals, 27, 1108-1114. http://dx.doi.org/10.1016/j.chaos.2005.04.082

[367] Iovane, G. (2007) Chaos, Solitons \& Fractals, 31, 1041-1053. http://dx.doi.org/10.1016/j.chaos.2006.03.109

[368] Argyris, J., Ciubotariu, C. and Andreadis, I. (1998) Chaos, Solitons \& Fractals, 9, 16511701. http://dx.doi.org/10.1016/S0960-0779(97)00193-8

[369] Agop, M., Ioannou, P.D., Luchian, D., Nica, P., Radu, C. and Condurache, D. (2004) Chaos, Solitons \& Fractals, 21, 515-536. http://dx.doi.org/10.1016/j.chaos.2003.12.053

[370] Rossler, O.E., Fröhlich, D., Kleiner, N., Pfaff, M. and Argyris, J. (2004) Chaos, Solitons \& Fractals, 20, 205-208. http://dx.doi.org/10.1016/S0960-0779(03)00358-8

[371] Agop, M., Ioannou, P.D., Coman, P., Ciobanu, B. and Nica, P. (2001) Chaos, Solitons \& Fractals, 12, 1947-1982. http://dx.doi.org/10.1016/S0960-0779(00)00161-2

[372] Mukhamedov, A.M. (2007) Chaos, Solitons \& Fractals, 33, 717-724. http://dx.doi.org/10.1016/j.chaos.2006.11.016

[373] Harabagiu, A., Niculescu, O., Colotin, M., Bibire, T.D., Gottlieb, I. and Agop, M. (2009) Romanian Reports in Physics, 61, 395-400.

[374] Ord, G.N. (1999) Chaos, Solitons \& Fractals, 10, 499-512. http://dx.doi.org/10.1016/S0960-0779(98)00255-0

[375] Castro, C. and Granik, A. (2000) Chaos, Solitons \& Fractals, 11, 2167-2178. http://dx.doi.org/10.1016/S0960-0779(00)00027-8 
[376] Castro, C. (2002) Chaos, Solitons \& Fractals, 13, 203-207. http://dx.doi.org/10.1016/S0960-0779(00)00268-X

[377] Ahmed, N. (2004) Chaos, Solitons \& Fractals, 21, 773-781. http://dx.doi.org/10.1016/j.chaos.2004.01.013

[378] Sidharth, B.G. (2001) Chaos, Solitons \& Fractals, 12, 2357-2361. http://dx.doi.org/10.1016/S0960-0779(00)00182-X

[379] El Naschie, M.S. (2008) Chaos, Solitons \& Fractals, 36, 819-822. http://dx.doi.org/10.1016/j.chaos.2007.09.020

[380] El Naschie, M.S. (2008) Chaos, Solitons \& Fractals, 36, 819-822. http://dx.doi.org/10.1016/j.chaos.2007.09.020

[381] El Naschie, M.S. (2008) Chaos, Solitons \& Fractals, 36, 546-549. http://dx.doi.org/10.1016/j.chaos.2007.09.017

[382] Fred, Y.Y. (2009) Chaos, Solitons \& Fractals, 41, 2301-2305. http://dx.doi.org/10.1016/i.chaos.2008.09.004

[383] El-Okaby, A.A. (2008) Chaos, Solitons \& Fractals, 38, 1305-1317. http://dx.doi.org/10.1016/j.chaos.2008.02.034

[384] Sidharth, B. and Altaisky, M.V. (Eds.) (2012) Frontiers of Fundamental Physics 4. Springer Science \& Business Media.

[385] Buzea, C.G., Agop, M., Galusca, G., Vizureanu, P. and Ionita, I. (2007) Chaos, Solitons \& Fractals, 34, 1060-1074. http://dx.doi.org/10.1016/j.chaos.2006.03.122

[386] El Naschie, M.S. (2005) Chaos, Solitons \& Fractals, 23, 683- 688. http://dx.doi.org/10.1016/j.chaos.2004.06.032

[387] El Naschie, M.S. (2005) Chaos, Solitons \& Fractals, 23, 683- 688. http://dx.doi.org/10.1016/j.chaos.2004.06.032

[388] Ahmed, E. and Hegazi, A.S. (2000) Chaos, Solitons \& Fractals, 11, 1759-1761. http://dx.doi.org/10.1016/S0960-0779(99)00082-X

[389] Tomaschitz, R. (1997) Fractals, 5, 215-220. http://dx.doi.org/10.1142/S0218348X97000206

[390] Castro, C. (2000) Foundations of Physics, 30, 1301-1316. http://dx.doi.org/10.1023/A:1003640606529

[391] Christianto, V. (2003) Apeiron, 10, 231.

[392] Colotin, M., Niculescu, O., Bibire, T. D., Gottlieb, I., Nica, P. and Agop, M. (2009) Romanian Reports in Physics, 61, 387-394.

[393] Dariescu, C., Dariescu, M.A. and Murariu, G. (2007) Chaos, Solitons \& Fractals, 34, 10301036. http://dx.doi.org/10.1016/j.chaos.2006.04.070

[394] Agop, M., Jarcau, M. and Stroe, A. (2005) Chaos, Solitons \& Fractals, 25, 781-790. http://dx.doi.org/10.1016/j.chaos.2004.12.036

[395] Lorenzi, M.G., Francaviglia, M. and Iovane, G. (2008) APLIMAT-Journal of Applied Mathematics, 1, 109-119.

[396] He, J.H., Liu, Y., Mo, L.F., Wan, Y.Q. and Xu, L. (2008) Electrospun Nanofibres and Their Applications. iSmithers, Shawbury, Shrewsbury, Shropshire, UK.

[397] Yildiz, A., De, U.C. and Cetinkaya, A. (2013) Proceedings of the National Academy of Sciences, India Section A: Physical Sciences, 83, 239-245.

http://dx.doi.org/10.1007/s40010-013-0071-y

[398] Sidharth, B.G. (2002) Chaos, Solitons \& Fractals, 14, 1325-1330. 
http://dx.doi.org/10.1016/S0960-0779(02)00085-1

[399] Ord, G.N. and Gualtieri, J.A. (1998) Biosystems, 46, 21-28. http://dx.doi.org/10.1016/S0303-2647(97)00077-4

[400] Zhong, T. and He, J.H. (2013) Fractal Spacetime and Non-Commutative Geometry in Quantum and High Energy Physics, 3, 46-49.

[401] Dariescu, C. and Dariescu, M.A. (2007) Chaos, Solitons \& Fractals, 32, 8-14. http://dx.doi.org/10.1016/j.chaos.2006.05.042

[402] Sidharth, B.G. (2002) Chaos, Solitons \& Fractals, 14, 525-527. http://dx.doi.org/10.1016/S0960-0779(01)00197-7

[403] Agop, M., Oprea, I., Sandu, C., Vlad, R., Buzea, C.G. and Matsuzawa, H. (2000) Australian Journal of Physics, 53, 231-240.

[404] Castro, C. and Granik, A. (2000) On M Theory, Quantum Paradoxes and the New Relativity. arXiv preprint physics/0002019.

[405] El Naschie, M.S. (2005) Chaos, Solitons \& Fractals, 25, 911-913. http://dx.doi.org/10.1016/j.chaos.2004.12.002

[406] Meissner, W. (1996) Chaos, Solitons \& Fractals, 7, 697-709. http://dx.doi.org/10.1016/0960-0779(94)00220-7

[407] Buzea, C.G., Rusu, I., Bulancea, V., Bădărău, G., Păun, V.P. and Agop, M. (2010) Physics Letters A, 374, 2757-2765. http://dx.doi.org/10.1016/j.physleta.2010.04.044

[408] Cristescu, C.P., Mereu, B., Stan, C. and Agop, M. (2009) Chaos, Solitons \& Fractals, 40, 975-980. http://dx.doi.org/10.1016/j.chaos.2007.08.054

[409] Penrose, R. (2004) The Road to Reality. A Complete Guide to the Laws of the Universe. Jonathan Cape, London.

[410] Green, B. (2004) The Fabric of the Cosmos. Penguin Books, London.

[411] Hawkings, S and Ellis, G. (1973) The Large Scale Structure of Space-Time. Cambridge University Press, Cambridge. http://dx.doi.org/10.1017/CBO9780511524646

[412] 'tHooft, G. (1997) In Search of the Ultimate Building Blocks. Cambridge University Press, Cambridge.

[413] Davies, P. (Editor) (1989) The New Physics. Cambridge University Press, Cambridge.

[414] Halpern, P. (2004) The Great Beyond. John Wiley, New Jersey.

[415] Zeilinger, A. (2003) Einstein’s Schleier. C. H. Beck Verlog, Munchen.

[416] Crowell, L.B. (2005) Quantum Fluctuations of Spacetime. World Scientific, Singapore. http://dx.doi.org/10.1142/5952

[417] Smolin, L. (2000) Three Roads to Quantum Gravity. Weidenfeld and Nicolson, London.

[418] 'tHooft, G. (1994) Under the Spell of the Gauge Principle. World Scientific, Singapore.

[419] Kaku, M. (2000) Strings, Conformal fields and M-Theory. Springer, New York. http://dx.doi.org/10.1007/978-1-4612-0503-6

[420] Connes, A. (1994) Non-Commutative Geometry. Academic Press, San Diego.

[421] Becker, K., Becker, M. and Schwarz, J. (2007) String Theory and M-Theory. Cambridge University Press, Cambridge.

[422] Green, M., Schwarz, J. and Witten, E. (1987) Superstring Theory. Vol. I and II. Cambridge University Press, Cambridge.

[423] El Naschie, M.S. (2016) Journal of Modern Physics, 7, 1953-1962. http://dx.doi.org/10.4236/jmp.2016.714173 
[424] Weinberg, S. (1995) The Quantum Theory of Fields. Vol. I. Cambridge University Press, Cambridge. http://dx.doi.org/10.1017/CBO9781139644167

[425] Oriti, D. (Editor) (2009) Approaches to Quantum Gravity. Cambridge University Press, Cambridge.

[426] Quoting Einstein. (2013) www.blogspot.com/2013/06/einstein-and-michele-besso.html

[427] Einstein, A. (1989) Letter to Michele Besso's Family. Ref. Bernstein, Jeremy. A Critic at Large: Besso. The New Yorker.

Submit or recommend next manuscript to SCIRP and we will provide best service for you:

Accepting pre-submission inquiries through Email, Facebook, LinkedIn, Twitter, etc. A wide selection of journals (inclusive of 9 subjects, more than 200 journals)

Providing 24-hour high-quality service

User-friendly online submission system

Fair and swift peer-review system

Efficient typesetting and proofreading procedure

Display of the result of downloads and visits, as well as the number of cited articles

Maximum dissemination of your research work

Submit your manuscript at: http://papersubmission.scirp.org/

Or contact jmp@scirp.org 